\title{
A multi-agent based micro-grid operation method considering charging and discharging strategies of electric vehicles
}

\author{
Takeshi Nagata ${ }^{a}$, Shogo Monde ${ }^{a^{*}}$ \\ ${ }^{a}$ Hiroshima Institute of Technology, Hiroshima, Japan
}

\begin{abstract}
In this paper, we propose a multi-agent based micro-grid (MG) operation method considering charging and discharging electric vehicles (EVs). The proposed system consists of five types of agent: Micro Grid controller agent (MGC), Load agents (LAGs), Gas-turbines/engine agents (GAGs), Photovoltaic generation agents (PVAGs), and Electric Vehicle agents (EVAGs). In the proposed method, the load balancing can be realized by suppressing sudden fluctuations in supply and demand balance due to the synchronization of charging and discharging of EVs. From the simulation results, it can be seen the proposed multi-agent system could realize the load equalization in MG.
\end{abstract}

Keywords: Microgrids, electric vehicles, charge and discharge control, multi-agent, mixed integer programming

\section{Introduction}

Today, the introduction of renewable energy source (RES) is advanced worldwide to realize a lowcarbon society. According to the International Energy Agency (IEA), the introduction volume of PV in Japan is 7.80 million $\mathrm{kW}$ in 2016 and the total generation capacity is 42.40 million $\mathrm{kW}$ [1]. It is pointed out that such RES has various influences on the electric power system since the output fluctuation due to the weather is large. As one of these countermeasures, expectations for the use of storage batteries including electric vehicles (EVs) are increasing [2,3], and a number of studies are being conducted. Typical ones are those related to frequency adjustment [4, 5], voltage management [6], expenses such as operating cost and income of EV owners [7, 8], and load leveling [9-13].

In this research, we focus on the load leveling by EV. If a large amount of EV is introduced in the future and it is connected to the distribution system, a large electric power demand (charging power) will be newly generated from the grid side. Also, if the discharge power is utilized, the EV functions as a distributed power supply. When such charge and discharge operations of the EV are performed synchronously, there is concern that large fluctuations in supply-demand balance may occur in the distribution system.

Reference [12] is a centralized system for determining the optimum charging strategy to suppress power peaks, while [13] is a distributed system for EV charging control by using multi-agents approach. However, these studies are limited to unidirectional V2G, which treats EV as a controllable load. Therefore, in this research, we consider the feeder of the distribution system as a microgrid (MG) with elements such as load, gas engine generator, photovoltaic generator and EV, and propose a MG operation multi-agent system considering charge and discharge of EV control.

The features of this proposed method are as follows.

- In the proposed method, load balancing can be realized by suppressing sudden fluctuations in supplydemand balance due to synchronization of charging and discharging of EVs.

- In order to avoid synchronization of EV control, the proposed system monitors the EV charging rate

\footnotetext{
* Manuscript received May 31, 2018; revised January 16, 2019.

Corresponding author. Tel.: +81-82-921-4367; E-mail address: t.nagata.wp@it-hiroshima.ac.jp.

doi: $10.12720 /$ sgce.8.2.149-155
} 
(SOC: state of charge) at regular time intervals (for example, every 60 minutes), and determines the "Charging EV candidate list (in ascending order of SOC)" and the "Discharging EV candidate list (in descending order of SOC)". Then, based on the maximum demand forecast value, the number of parallelizable charging or discharging can be determined, and the excessive synchronization is avoided.

- Also, by monitoring the active power of the distribution line feeder of the distribution substation, the EV control according to the supply-demand balance is performed. That is, when surplus electric power is generated such as when the weather is fine on a holiday, the discharge of EV is suppressed and charging of the EV in daytime is promoted.

This proposed system was implemented with Java language, and simulations were performed on a model system. The results showed that flexible microgrid operation was realized.

\section{Mathematical Formulation of Microgrid Operation}

In this paper, a feeder of the distribution system is regarded as a MG and the purpose of load leveling of the MG is aimed.

\subsection{Mathematical formulation of the microgrid operation}

Optimum operation plan of a microgrid can be formulated as a mixed integer programming problem of minimizing the total power generation cost of distributed power and power purchase cost over a discussion period $(i=1 \ldots \mathrm{T})$. In the proposed method, we formulate the optimal MG operation problem as an optimization problem of each time as follows.

1. Objective function

$$
\min \left\{E P(t) \cdot P(t)+F P(t) \sum_{i=1}^{I} G_{i}(t)\right\}
$$

In equation (1), 1 st term is an electric power purchase cost from a grid. Note that it also includes cost for electrical demand as well as charging to EVs. 2nd term is power generation cost of gas engines. Startup or shutdown of a gas engine is determined on balance with the power expense from the grid. Where, $\mathrm{EP}(\mathrm{t})$ : electricity rate at time $\mathrm{t}, \mathrm{P}(\mathrm{t})$ : purchase power from grid at time $t, \mathrm{FP}(\mathrm{t})$ : generation rate of gas engine at time $t, \mathrm{G}_{\mathrm{i}}(\mathrm{t})$ : output of gas engine i generator at time $\mathrm{t}$, $\mathrm{I}$ : total number of gas engine.

\section{Constraints}

There are following constraints in the microgrid operation.

(a) Supply-and-demand balance conditions

Power supply and demand in a microgrind must balance.

$$
P(t)+\sum_{i=1}^{I} G_{i}(t)+\sum_{j=1}^{J}\left(B_{j}(t)-C_{j}(t)\right)+\sum_{k=1}^{K} P V_{k}(t)+\sum_{\ell=1}^{L} W T_{\ell}(t)=D(t)
$$

where, $B_{j}(t)$ : discharge power of EV $j$ at time $t, C_{j}(t)$ : charging power to $E V j$ at time $t, P_{k}(t)$ : power output of PV k at time t, $\mathrm{D}(\mathrm{t})$ : power demand at time $\mathrm{t}$, J: total number of EVs, K: total number of PVs.

(b) Generator output upper and lower limit constraints

Upper and lower limits exist in a power generation output of a gas engine.

$$
G_{i}^{\min } \cdot x_{i}(t) \leq G_{i}(t) \leq G_{i}^{\max } \cdot x_{i}(t)
$$

where, $G_{i}{ }^{m i n}$ : minimum output of gas engine $i, G_{i}{ }^{\max }$ : maximum output of gas engine $i, x_{i}(t)$ : decision variable of startup or shutdown of the gas engine at time t (0: shutdown, 1: startup). 
(c) Generator minimum down-time constrains

A gas engine cannot be started for a certain fixed period after stopping in order to reduce the number of times of startup.

$$
x_{i}(t-1)-x_{i}(t) \leq\left(1-x_{i}(\tau)\right) \quad\left(\tau=t+1, \cdots, \min \left(t+D T^{\min }-1, T\right)\right)
$$

where, $\mathrm{DTi}^{\mathrm{min}}$ : minimum downtime of gas engine $\mathrm{i}, \mathrm{T}$ : total number of hours.

(d) Generator minimum up-time constraints

A gas engine cannot be stopped for a certain fixed period after starting in order to reduce the number of stops.

$$
x_{i}(t)-x_{i}(t-1) \leq x_{i}(\tau)\left(\tau=t+1, \cdots, \min \left(t+U T^{\min }-1, T\right)\right)
$$

where, $\mathrm{UT}^{\mathrm{min}}$ : minimum uptime of gas engine $\mathrm{i}$.

(e) Storage EV operation constraints

An operation of a EV is either "discharge" or "charge" or "halt".

$$
y_{j}(t)+z_{j}(t) \leq 1
$$

where, $\mathrm{y}_{\mathrm{j}}(\mathrm{t})$ : decision variable of discharge of $\mathrm{EV} \mathrm{j}$ at time $\mathrm{t}\left(0\right.$ : halt, 1 : discharge), $\mathrm{z}_{\mathrm{j}}(\mathrm{t})$ : decision variable of charge of $\mathrm{EV}_{\mathrm{j}}$ at time $\mathrm{t}$ (0: halt, 1: charge).

(f) Storage EV charge capacity constrains

$$
C_{j}^{\min } \leq C_{j}(0)+\sum_{\gamma=1}^{t}\left(C_{j}(\gamma)-B_{j}(\gamma)\right) \leq C_{j}^{\max }
$$

where, $\mathrm{C}_{\mathrm{j}}^{\text {min }}$ : minimum capacity of $\mathrm{EV} \mathrm{j}, \mathrm{C}_{\mathrm{j}}^{\text {max }}$ : maximum capacity of $\mathrm{EV}_{\mathrm{j}}, \mathrm{Cj}(0)$ : initial capacity of $\mathrm{EV} \mathrm{j}$.

(g) Storage EV ramp rate constraints

An EV has ramp-up and ramp-down rates.

$$
\begin{aligned}
& 0 \leq C_{j}(t) \leq d C_{j}^{\max } \cdot \Delta t \cdot z_{j}(t) \\
& 0 \leq B_{j}(t) \leq d B_{j}^{\max } \cdot \Delta t \cdot y_{j}(t)
\end{aligned}
$$

where, $\mathrm{dC}_{\mathrm{j}}^{\text {max }}$ : maximum charging ramp rate of $\mathrm{EV} \mathrm{j}, \mathrm{dB}_{\mathrm{j}}{ }^{\max }$ : maximum discharging ramp rate of $\mathrm{EV} \mathrm{j}, \Delta \mathrm{t}$ : time interval.

(h) Must-charge constraints

This is a constraint to charge an EV. This constraint can be used as a microgrid operation strategy to charge in the time frame tc in preparation for a discharge in the future. In general, an EV is charged in a cheap mid-night hours of electricity rate.

$$
z_{j}\left(t_{c}\right)=1
$$

(i) Must-discharge constraints

This is a constraint to discharge an EV. This constraint is used as a microgrid operation strategy to be discharged at the time frame td.

$$
y_{j}\left(t_{d}\right)=1
$$




\subsection{EV charging and discharging strategies}

Fig. 1 shows the EV charging and discharging determination algorithm. First, the active power flowing into the MG from the SS and the SOC of the EV are measured at constant time intervals. Then, if the time is the charging period, a "charging EV candidate list (C-List)" in which each EV is rearranged in ascending order of SOC is created. Next, as shown in equation (11), the number of EVs that can be charged in parallel is obtained under the condition that the charging power of the parallel EV at that time is not more than $\alpha$ times the maximum demand $(\alpha \leq 1.0)$.

$$
\sum_{n c=1}^{N C} d C_{n c}^{\max } \leq \alpha \cdot D^{\max }
$$

Then, the decision variables of C-List are set to $z_{n c}(t)=1$.

If the time is a discharge period and no surplus electric power is generated, a "discharge EV candidate list (D-List)" in which each EV is rearranged in descending order of SOC is created. Then, as shown in the equation (12), the number of EV units that can be discharged in parallel is obtained under the condition that the discharge electric power of the parallel EV at that time is not more than $\alpha$ times the maximum demand $(\alpha \leq 1.0)$.

$$
\sum_{n d=1}^{N D} d B_{n d}^{\max } \leq \alpha \cdot D^{\max }
$$

\section{Proposed Multi-agent Microgrid Operation}

\subsection{Overview of the proposed system}

An agent model of a microgrid is shown in Fig. 2. As shown in this figure, the proposed system consists of five kinds of agents (microgrid controller (MGC); load agent (LAG); generator agent (GAG); photovoltaic agent (PVAG); EV agent (EVAG)).

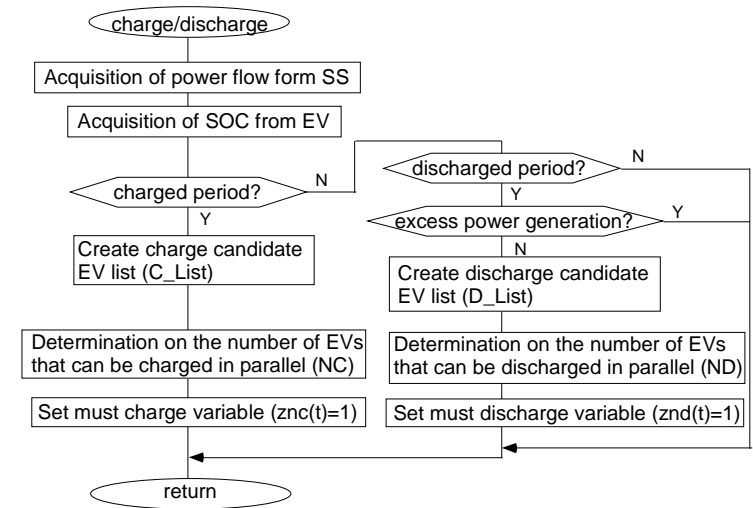

Fig. 1. EV charging and discharging determination algorithm.

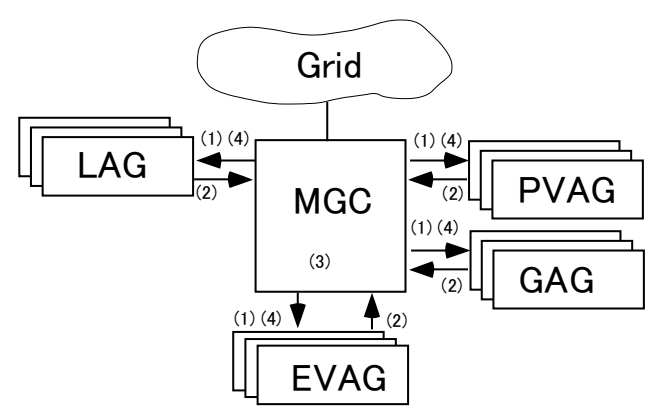

Fig. 2. Microgrid agent model.

\subsection{Processing of microgrid controller}

In this section, we explain the processing of MGC which plays an important role in the proposed system. In the proposed system, it is implemented as a state transition machine with Java classes as states. The MGC is composed of four states S0 to S3. First, the state S0 is an initialization processing. The simulation model file is read at first, parameters are transmitted to each agent (LAGs, PVAGs, GAGs, 
EVAGs), and the number of simulation steps is initialized. Next, the state $\mathrm{S} 1$ is a process of collecting environmental information. In order to collect the information of this step, MGC asks each AG to submit the value of load and/or generated power. MGC also collects the inflow power from SS and SOCs. The state S2 is a decision making process. Here, after collecting information from each AG, the charging and discharging strategies of EVs in Section 2.1 is applied. In order to solve the mixed integer programming problem shown in equations (1) - (10), GLPK [14] is used. Next, state S3 is a result report (control signal output) process. The decision result of the optimization problem is transmitted to each AG. Then, the completion of the simulation is judged. If the simulation is continued, the number of steps is incremented and the state transitions to the state S1. Otherwise, the process ends.

\section{Simulation}

\subsection{Simulation conditions}

In order to confirm the basic operation of the agent, a MG model including EVAGs, GAGs, PVAGs, and LAG was used. Table 1 shows parameters of each element, electricity price (EP), and gas engine power generation fee (FP). One EVAG is assumed to be composed of 120 electric cars with a capacity of $40(\mathrm{kWh})$ and the capacity would be $4,800(\mathrm{kWh})$. The demand and photovoltaic power generation were created based on our university actual measurement data. The demand ranges from about 500 to 1,500 $\mathrm{kW}$. As shown in this table, EP was set by time zone with reference to electric company's price, and FP was fixed. In addition, the time interval of MGC task announcement was set to 10 minutes, and 24 hour simulation was performed every 10 minutes.

In order to carry out the charging time zone of EV at inexpensive EP from 23:00 to 7:00, many simulations for 2 days operation was carried out. Table 2 shows two case studies described below. As shown in this table we have set up a distinction between weekdays and holidays and weather. It is assumed that the load is heavy on weekdays and solar photovoltaic power would increase on sunny days.

\subsection{Case-1(day 1: weekday and fine day; day 2: weekday and fine day)}

The result of Case-1 is shown in Fig. 3. Here, WF - WF indicates that the first day is weekday and fine, and the second day is weekday and fine weather. In this figure, the vertical axis shows the generated power (including inflow power from the grid) on the plus side and the load power on the minus side. Referring to the case without control in Fig. 3 (a), it can be seen that a large change occurs in the active power flowing into the MG due to the synchronous charging and discharging. On the other hand, in the case with control in Fig. 3 (b), it can be seen that the synchronous EV operation is suppressed. In this case, the load factor for 2 days has been improved from $52.6 \%$ to $68.9 \%$.

\subsection{Case-2(day 1: holiday and fine; day 2: weekday and rainy)}

The result of Case-2 is shown in Fig. 4. Here, the HF - WR indicates that the first day is a holiday and fine, and the second day is weekday and rainy. In the without control case, though the results are not described due to space limitation, the reverse power flow occurred due to the large photovoltaic power generation at the light load in the daytime, because it is a holiday and fine day on the first day. In the case with the control in Fig. 4 (a), it can be seen that the daytime discharge in the day is suppressed by the charging and discharge strategies of EVs in Section 2.1, and no reverse flow has occurred. Then the charging electricity of EV at midnight on the first day is discharged in the daytime on the second day of rainy weather. As a result, the load factor for 2 days has improved from $40.8 \%$ in the case without control to $49.9 \%$. Furthermore, Fig. 4 (b) is the result of the case assuming that the MG has gas engine generators. By starting gas engines, the load factor for 2 days has been further improved to $58.7 \%$. It can also be considered that the shortage of the photovoltaic power generated in the rainy weather is backed up by both the EV discharging and the gas engine generators. 


\section{Conclusions}

In this paper, we consider the distribution line feeder in which a large amount of EV and RES are interconnected, and propose a microgrid operation method considering the charge and discharge control of EVs. Although it is a simulation with a simple microgrid model, the load leveling function is realized by incorporating the charging and discharging strategies of EVs in the microgrid controller. In addition, it is considered that the proposed method has the possibility to autonomous operation of the microgrid by monitoring the active power of the distribution line feeder and the SOC of EVs. Future tasks include examinations from the viewpoint of practical use, such as collection of SOC data of a large amount of EVs and control of voltage and frequency fluctuation.

Table 1. Simulation parameters

\begin{tabular}{ll|ll}
\hline Parameter & Value & Parameter & Value \\
\hline Peak load $(\mathrm{kW})$ & 1,500 & $\mathrm{EV}(\mathrm{kWh})$ & $\mathrm{C}^{\min }=480, \mathrm{C}^{\max }=4,800$ \\
GAG $(\mathrm{kW})$ & $\mathrm{G}^{\min }=20, \mathrm{G}^{\max }=200$ & $\mathrm{EP}($ Yen $/ \mathrm{kWh})$ & $\mathrm{Night}=10.0$, Day=30.0 \\
UTmin, DTmin $(\mathrm{h})$ & 2,2 & FP $($ Yen $/ \mathrm{kWh})$ & 28.0 \\
PV $(\mathrm{kW})$ & 200 & Dt $(\min )$ & 10 \\
\hline
\end{tabular}

Table 2. Case studies

\begin{tabular}{l|ll|ll}
\hline \multirow{2}{*}{ Case } & Day 1 & & Day 2 & \\
\cline { 2 - 5 } & Type & Weather & Type & Weather \\
\hline 1 & weekday & fine & weekday & fine \\
2 & holiday & fine & weekday & rainy \\
\hline
\end{tabular}

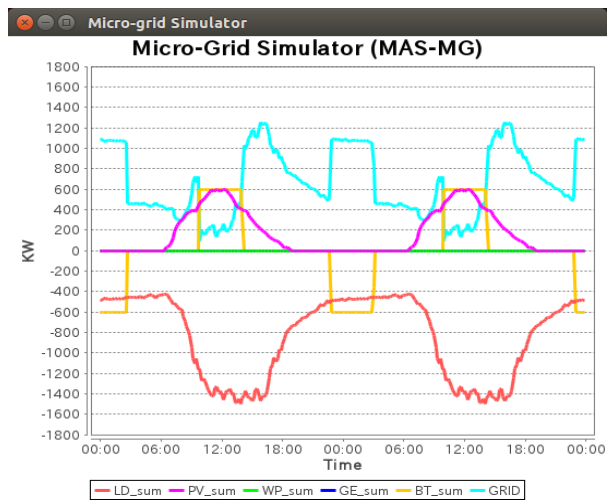

(a) Without control (WF-WF)

Fig. 3. Case-1 (WF-WF).

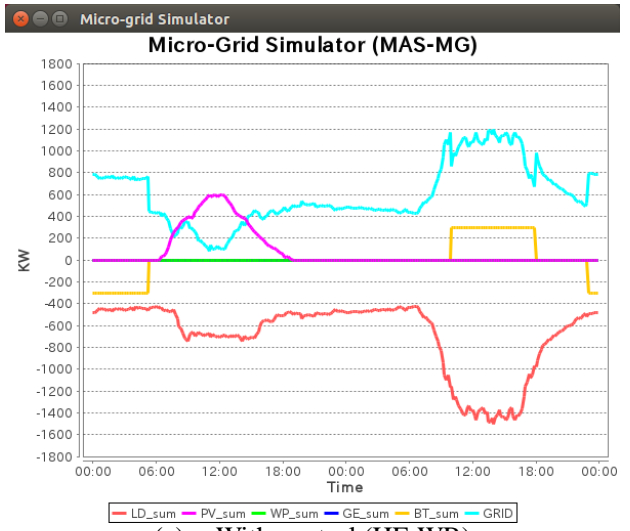

(a) With control (HF-WR)

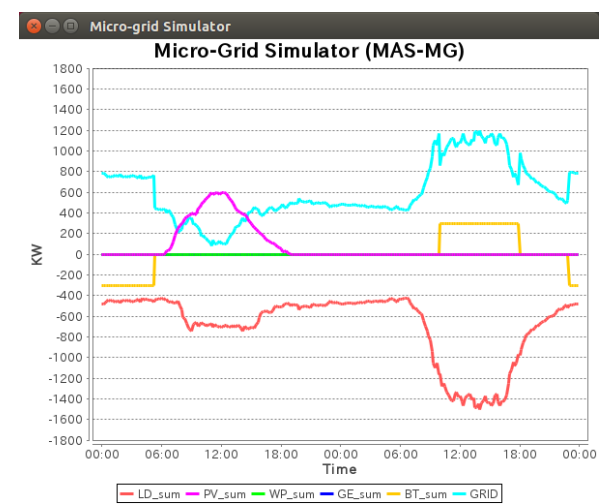

(b) With control(WF-WF)

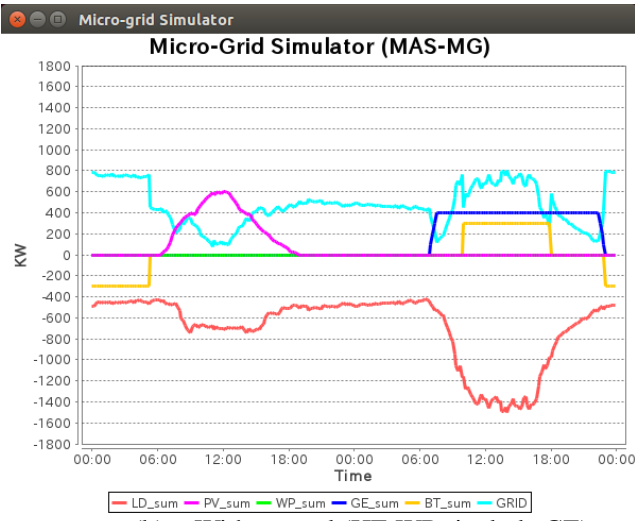

(b) With control (HF-WR, include GE)

Fig. 4. Case-2 (HF-WR). 


\section{References}

[1] International Energy Agency (IEA), [Online]. Available: http://www.iea-pvps.org

[2] Ota Y. Cooperation between Power Systems and Electric Vehicles. IEEJ Transaction on Power and Energy, 2017; 133(6):497-500.

[3] Fukushima S. Trends of application and control technology of battery energy storage for power systems. IEEJ Transaction on Power and Energy, 2017; 137(10): 644-647.

[4] Masuda S, Shimizu K, Yokoyama A. Load frequency control by use of a number of both heat pump water heaters and electric vehicles in power systems with a large integration of renewable energy sources. IEEJ Transaction on Power and Energy, 2017; 132(1):23-33.

[5] Hosogoe H, Mitsumoto M, Tobita Y, Takaguchi Y, Mitani T. Development of frequency control method by large scale Battery energy storage system. IEEJ Transaction on Power and Energy, 2017; 137(10):648-654.

[6] Nagashima H, Kajiwara A, Iihoshi K, Kai Y. Supply and demand balance improvement using 300 MWh-class storage system. IEEJ Transaction on Power and Energy, 2017; 137(10):671-677.

[7] Papadopoulos P, Jenkis N, Cipcgan L. M, Grau I, Zabala E. Coordinatipn of the charging of electric vehicles using a multiagent system, IEEE Transactions on Smart Grid, 2013; 4(4): 1802-1809.

[8] Shao C, Wang X. Shahidehpour M, Wang X, Wang B. Partial decomposition for distributed electric vehicle charging control considering electric power grid congestion. IEEE Transactions on Smart Grid, 2017; 8(1):75-83.

[9] Koyanagi $\mathrm{H}$, Uriu Y. Charging and discharging time control of electric vehicles for the purpose of load leveling. IEEJ Transaction on Power and Energy, 1998; 118-B(5): 505-510.

[10] Takagi M, Iwafune Y, Yamamoto H, Yamaji K, Okano K, Hiwatari R, Ikeya T. Algorithm for bottom charge based on loadduration curve of plug-in hybrid electric vehicles. IEEJ Transaction on Power and Energy, 2010; 130(8):723-746.

[11] Ikegami T, Yano H, Kudo K, Ogimoto K. Effects of smart charging of multiple electric vehicles. IEEJ Transaction on Power and Energy, 2013; 133(6): 562-574.

[12] Sortomme E, El-Sharkwai MA. Optimal charging strategies for unidirectional vehicle-to-grid. IEEE Transactions on Smart Grid, 2011; 2(1):131-138.

[13] Karfopoulos EL, Hatziargyriou ND. A multi-agent system for controlled charging of a large population of electric vehicles. IEEE Transactions on Smart Grid, 2013;28,(2):,75-83.

[14] GLPK, [Online]. Available: https://www.gnu.org/software/glpk. 